\title{
Paper
}

\section{Generating Face Images Based on Golden Ratios for Facial Beauty}

\author{
Ran PenG* Non-member, Kohei InOUE ${ }^{\dagger *}$ Member \\ Kenji HARA* Member
}

(Received June 8, 2021, revised December 24, 2021)

\begin{abstract}
Golden ratio is considered an index of beauty in both natural and artificial objects. In this paper, we propose a method for generating face images based on the golden ratios discovered so far for facial beauty. For example, we focus on some current golden ratios for facial beauty, where we solve underdetermined systems by introducing additional and reasonable equations. Experimental results showed that the proposed method generates natural face images based on user inputs. We also evaluated the beauty of the generated face images objectively and subjectively, then observed that the proposed method improved the evaluation scores on average for both objective and subjective evaluations.
\end{abstract}

Keywords: Face image, Golden ratio, Facial beauty, Underdetermined system

\section{Introduction}

Human faces play a role of signboards which inform their features, conditions and feelings to people around them, and are helpful for smooth communication between people as suggested by the term "face-to-face communication" [1]. Therefore, people often wish to make their faces look as beautiful as possible. Laurentini and Bottino have surveyed 181 researches on the computer analysis of human beauty [2], where they referred to a number of beauty canons for human body and its parts.

In this paper, we focus on the golden ratios related to facial beauty. Mathematically, the golden ratio is defined as the ratio of $a$ to $b$ satisfying the equation $a: b=a+b: a$, and the golden number $\phi=a / b \approx 1.618$ is a solution to the quadratic equation $x^{2}-x-1=0$. However, the golden ratios for facial beauty are not restricted to this mathematical definition, and a variety of golden ratios have been proposed until now. Therefore, in this paper, we would like to use the term "golden ratio" as a generic term to refer to those ratios on facial beauty.

LAURIER PRESS [3] has introduced several golden ratios regarding the placement of parts of the face and the proportion of the vertical and horizontal lengths of it. Pallett et al. [4] tested the existence of an ideal facial feature arrangement that could optimize the attractiveness of any face given its facial features. Hatsui and Isaji [5] examined what are the factors of beautiful face in woman's university student, where a tool for generating a mean face [6] [7]. They generated two kinds of mean faces from two sets of face images, i.e., beautiful and not, where it was observed that the latter mean face became more beautiful than the original faces in the latter set.

\footnotetext{
$\dagger$ Corresponding: k-inoue@design.kyushu-u.ac.jp

* Department of Communication Design Science, Kyushu University

4-9-1, Shiobaru, Minami-ku, Fukuoka 815-8540, Japan
}

Recently, Leyvand et al. [8] have proposed a digital face beautification method based on the optimization of a beauty function modeled by support vector regressor called SVRbased beautifier. Diamant et al. [9] have shown the ability to generate realistic facial images conditioned on a beauty score using a variant of PGGAN (progressive growing of generative adversarial network) [10]. Those methods use face image datasets such as the AR face database [11] and CelebA [12] for semi-supervised training and deep learning, and realize reasonable beautification of face images.

In this paper, we propose a training data-free method for transforming a face image into another that matches specified golden ratios for facial beauty. We experimentally show the transformed faces from Japanese Female Facial Expression (JAFFE) Database [13], and evaluate them with web applications and a questionnaire survey.

The rest of this paper is organized as follows: Section 2 describes the proposed methods for several golden ratios for facial beauty. Section 3 shows the generated face images and the results of evaluations. Finally, Section 4 concludes this paper.

\section{Proposed Methods}

In this section, we describe three procedures for generating face images based on golden ratios for facial beauty.

2.1 Three Conditions for Beautiful Faces LAURIER PRESS [3] has introduced three conditions for beautiful faces as follows:

Condition 1 The ratios of the length of "hair line to inner ends of eyebrows", "inner ends of eyebrows to lower end of nose" and "lower end of nose to chin" are 1 : $1: 1$.

Condition 2 The width of a face is 5 times as long as the width of an eye. 
Condition 3 The ratio of the width and height of a face is $1: 1.46$.

To generate those face images satisfying the above conditions, we first present the original face image to a user. Then the user specifies the above "hair line", "inner ends of eyebrows", "lower end of nose" and "chin" on the presented image for Condition 1. Let $y_{0}, y_{1}, y_{2}$ and $y_{3}$ be the respective vertical coordinates with $y_{0}<y_{1}<y_{2}<y_{3}$, i.e., the vertical axis is expressed by a down-pointing arrow. Then the height of the face is given by $h=y_{3}-y_{0}$. Keeping the value of $h$ unchanged by fixing $y_{0}$ and $y_{3}$, we adjust $y_{1}$ and $y_{2}$ to the values that satisfy Condition 1 . Let $y_{0}^{\prime}, y_{1}^{\prime}, y_{2}^{\prime}$ and $y_{3}^{\prime}$ be the corresponding adjusted coordinates. Then we have $y_{0}^{\prime}=y_{0}, y_{3}^{\prime}=y_{3}$ and $\Delta h=y_{i+1}^{\prime}-y_{i}^{\prime}=\frac{h}{3}$ for $i=0,1,2$, from which we have $y_{i+1}^{\prime}=y_{i}^{\prime}+\frac{h}{3}$ recursively as $y_{1}^{\prime}=y_{0}+\frac{h}{3}$ and $y_{2}^{\prime}=y_{1}^{\prime}+\frac{h}{3}=y_{0}+\frac{2 h}{3}$. Next, we map a position $y \in\left[y_{i}, y_{i+1}\right]$ onto $y^{\prime} \in\left[y_{i}^{\prime}, y_{i+1}^{\prime}\right]$ by $\frac{y^{\prime}-y_{i}^{\prime}}{y-y_{i}}=\frac{\Delta h}{y_{i+1}-y_{i}}$ or $y^{\prime}=\frac{\Delta h}{y_{i+1}-y_{i}}\left(y-y_{i}\right)+y_{i}^{\prime}$ linearly.

Figure 1 shows an example of the generation of a face image satisfies Condition 1 from a face image in the Japanese Female Facial Expression Database (JAFFE) [13], which consists of 213 images of different facial expressions from 10 different Japanese female subjects. The red lines on the

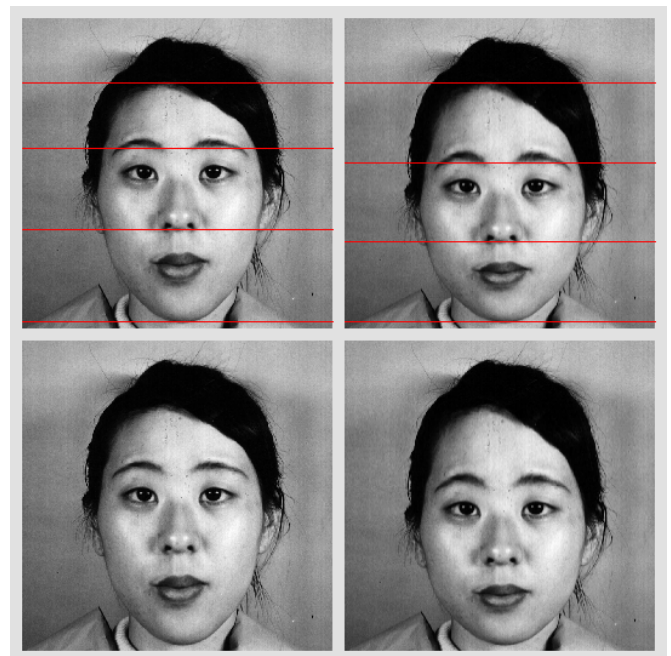

Figure 1: Generation of face image satisfying Condition 1: Left images show the original face, and right images show the transformed face, where red lines on the top images denote the user-specified (left) and the adjusted (right) positions of hair line, inner ends of eyebrows, lower end of nose and chin, from top to bottom. The bottom images are ones without red lines.

top left image show the vertical positions of "hair line", "inner ends of eyebrows", "lower end of nose" and "chin" specified by a user, and that on the top right image show the corresponding positions after the adjustment based on Condition 1. The bottom images show the red line-free input (left) and output (right) images. In this example, the forehead expands, and the area under the nose gets narrow.

Condition 2 demands that the width of an eye is a fifth of the width of the face. However, we cannot uniquely determine the position of eyes from Condition 2 only. To overcome this problem, we introduce an equal division of the width of a face into five regions as well as Prokopakis et al. [14], and place eyes at the second and fourth regions from left. Figure 2 shows the example for Condition 2, where the red lines from left to right in the top left image denote the left end of the face, the outer and inner corners of the right eye, the inner and outer corners of the left eye, and the right end of the face specified by a user. Let

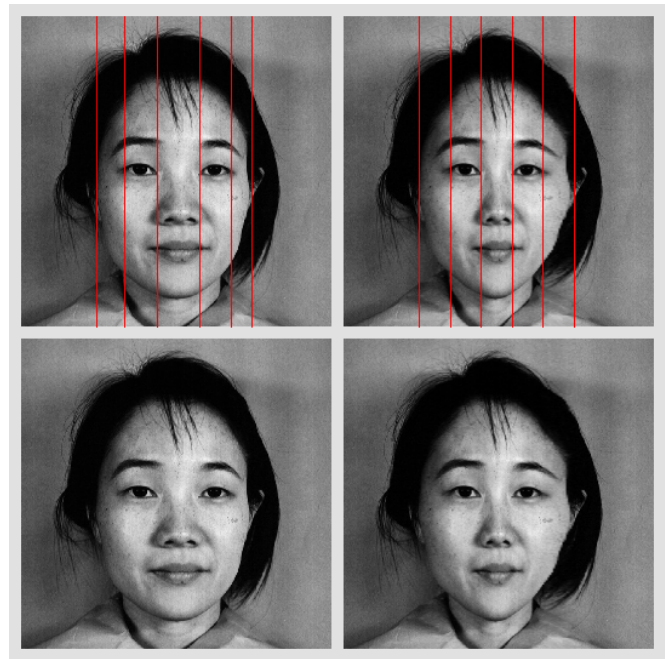

Figure 2: Generation of face image satisfying Condition 2, where the layout of images is the same as Figure 1. On the top left image, the left end of the face, the outer and inner corners of the right eye, the inner and outer corner of the left eye, and the right end of the face are indicated by red lines, which are placed at equal intervals on the top right image.

$x_{0}, x_{1}, \ldots, x_{5}$ be the respective horizontal coordinates with $x_{0}<x_{1}<\cdots<x_{5}$, i.e., the horizontal axis is expressed by a right arrow. Then the width of the face is given by $w=x_{5}-x_{0}$. Keeping the value of $w$ unchanged by fixing $x_{0}$ and $x_{5}$, we adjust $x_{1}, x_{2}, \ldots, x_{4}$ to the values that satisfy Condition 2 by the similar manner to the above one for Condition 1. In Figure 2 with the similar layout of images to Figure 1, the space between eyes gets narrower, and as a result, the nose and chin get thinner.

Condition 3 specifies the ratio of the width and height of a face. We would like to keep the height unchanged and adjust the width to satisfy Condition 3 . Figure 3 shows the example for Condition 3, where the user specifies hair line, chin, the left and right ends of the face as shown with red lines on the top left image. The top right image shows the transformed image where the width and height is adjusted so that the ratio coincide with $1: 1.46$. The resultant face in the bottom right image is slimmer than the original face in the bottom left image.

2.2 Pallett's Golden Ratios Pallett et al. [4] found that although different faces have varying attractiveness, individual attractiveness is optimized when the face's vertical distance between the eyes and the mouth is approximately 

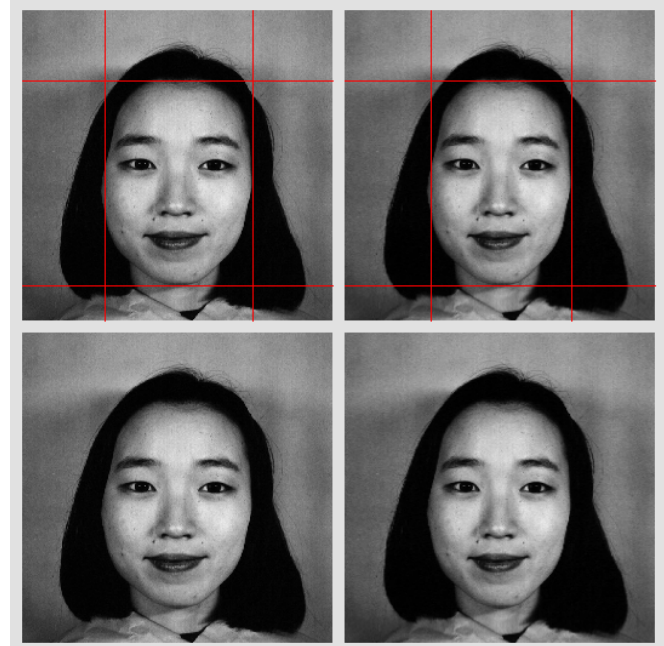

Figure 3: Generation of face image satisfying Condition 3, where the height of the face is preserved, and the width is adjusted in accordance with Condition 3.

$36 \%$ of its length, and the horizontal distance between the eyes is approximately $46 \%$ of the face's width. Figure 4 shows the intervals they focused on. In this subsection, we would like to use their results for beautifying face images.

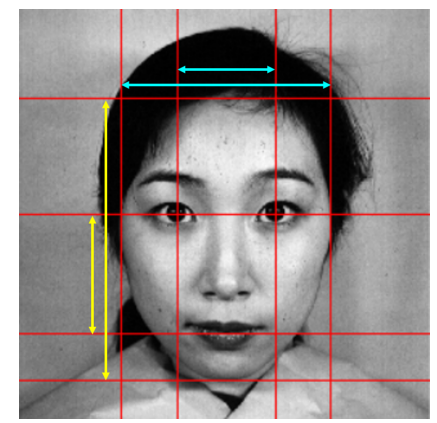

Figure 4: Intervals for Pallett's golden ratios.

Let $x_{0}, x_{1}, x_{2}$ and $x_{3}$ be the horizontal coordinates of the left end of a face, the centers of right and left eyes, and the right end of the face, respectively, and let $x_{0}^{\prime}=$ $x_{0}, x_{1}^{\prime}, x_{2}^{\prime}$ and $x_{3}^{\prime}=x_{3}$ be the corresponding adjusted coordinates. Then the Pallett's golden ratio can be expressed as $\left(x_{3}^{\prime}-x_{0}^{\prime}\right):\left(x_{2}^{\prime}-x_{1}^{\prime}\right)=1: 0.46$, where we want to know the values of $x_{1}^{\prime}$ and $x_{2}^{\prime}$. We assume that

$$
\begin{aligned}
& x_{1}^{\prime}=x_{1}-l \\
& x_{2}^{\prime}=x_{2}+r,
\end{aligned}
$$

and try to determine the values of $l$ and $r$, where if $l$ is positive, then $x_{1}^{\prime}$ moves to the left side of $x_{1}$, and if $r$ is positive, then $x_{2}^{\prime}$ moves to the right side of $x_{2}$. After the movement, we expect to have

$$
\frac{x_{2}^{\prime}-x_{1}^{\prime}}{x_{3}^{\prime}-x_{0}^{\prime}}=\frac{\left(x_{2}+r\right)-\left(x_{1}-l\right)}{x_{3}-x_{0}}=0.46,
$$

from which, however, we cannot determine the values of $l$ and $r$ uniquely. To overcome this problem, we introduce another equation as follows:

$$
\frac{l}{r}=\frac{x_{1}-x_{0}}{x_{3}-x_{2}}
$$

which means that the ratio of the variations in the left and right sides is equal to the ratio of their original distances. Solving (3) and (4) simultaneously for $l$ and $r$, we have

$$
\begin{aligned}
& l=\frac{0.46\left(x_{3}-x_{0}\right)-\left(x_{2}-x_{1}\right)}{\left(x_{3}-x_{0}\right)-\left(x_{2}-x_{1}\right)}\left(x_{1}-x_{0}\right) \\
& r=\frac{0.46\left(x_{3}-x_{0}\right)-\left(x_{2}-x_{1}\right)}{\left(x_{3}-x_{0}\right)-\left(x_{2}-x_{1}\right)}\left(x_{3}-x_{2}\right) .
\end{aligned}
$$

Substituting these values into (1) and (2), we have the target coordinates $x_{1}^{\prime}$ and $x_{2}^{\prime}$.

In the vertical direction, we specify the position of hair line, eyes, mouth and chin as $y_{0}, y_{1}, y_{2}$ and $y_{3}$, respectively. Let $y_{0}^{\prime}=y_{0}, y_{1}^{\prime}, \quad y_{2}^{\prime}$ and $y_{3}^{\prime}=y_{3}$ be the corresponding adjusted coordinates. Then the Pallett's golden ratio can be expressed as $\left(y_{3}^{\prime}-y_{0}^{\prime}\right):\left(y_{2}^{\prime}-y_{1}^{\prime}\right)=1: 0.36$, where we want to know the values of $y_{1}^{\prime}$ and $y_{2}^{\prime}$. We assume that

$$
\begin{aligned}
& y_{1}^{\prime}=y_{1}-t \\
& y_{2}^{\prime}=y_{2}+b,
\end{aligned}
$$

and try to determine the values of $t$ and $b$ as well as the above $l$ and $r$, where if $t$ is positive, then $y_{1}^{\prime}$ moves to the upper side of $y_{1}$, and if $b$ is positive, then $y_{2}^{\prime}$ moves to the lower side of $y_{2}$. After the movement, we expect to have

$$
\frac{y_{2}^{\prime}-y_{1}^{\prime}}{y_{3}^{\prime}-y_{0}^{\prime}}=\frac{\left(y_{2}+b\right)-\left(y_{1}-t\right)}{y_{3}-y_{0}}=0.36
$$

which is insufficient to determine the values of $t$ and $b$ uniquely. Therefore, we add the following equation:

$$
\frac{t}{b}=\frac{y_{1}-y_{0}}{y_{3}-y_{2}}
$$

which means that the ratio of the variations in the top and bottom sides is equal to the ratio of their original distances. Solving (9) and (10) simultaneously for $t$ and $b$, we have

$$
\begin{aligned}
& t=\frac{0.36\left(y_{3}-y_{0}\right)-\left(y_{2}-y_{1}\right)}{\left(y_{3}-y_{0}\right)-\left(y_{2}-y_{1}\right)}\left(y_{1}-y_{0}\right) \\
& b=\frac{0.36\left(y_{3}-y_{0}\right)-\left(y_{2}-y_{1}\right)}{\left(y_{3}-y_{0}\right)-\left(y_{2}-y_{1}\right)}\left(y_{3}-y_{2}\right) .
\end{aligned}
$$

Substituting these values into (7) and (8), we have the target coordinates $y_{1}^{\prime}$ and $y_{2}^{\prime}$.

2.3 Hatsui and Isaji's Golden Ratios Hatsui and Isaji [5] clarified the boundary line of a beautiful face and an ugly face, and found the factors of beautiful woman's face. According to their results, a beautiful face has the following properties: The width of an eye (the distance between the inner and outer corners of the eye) is $28 \%$ of the width of the face, and the distance between the inner corners of both eyes is $26 \%$ of the width of the face. these properties specify the percentage of the distance between the left (right) end of a face and the outer corner of the right (left) 


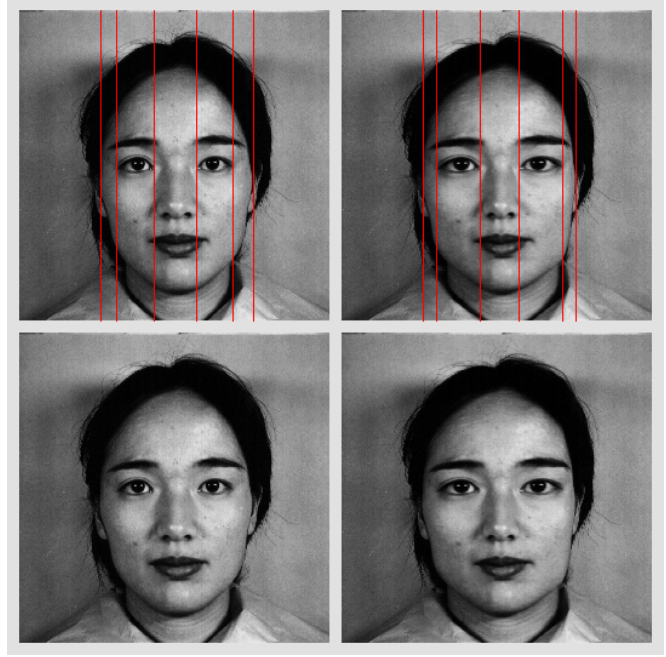

Figure 5: Horizontal transformation based on Hatsui and Isaji's golden ratios.

eye as $(100 \%-2 \times 28 \%-26 \%) / 2=9 \%$. Figure 5 shows an example of the generation of a face image based on Hatsui and Isaji's golden ratios, where the eyes become larger, and accordingly, the forehead and lower jaw expand.

In addition to the above horizontal transformation, we combine the vertical one based on the golden ratios introduced by Laurentini and Bottino [2] as follows: Figure 6 shows the golden ratios for vertical transformation, where

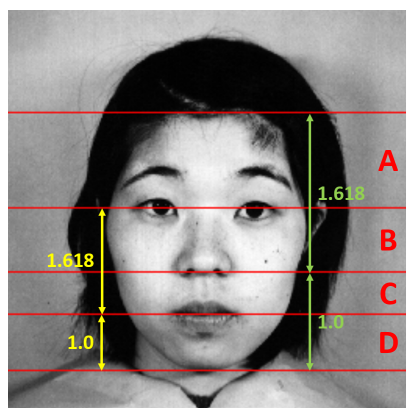

Figure 6: Golden ratios in face.

we can see two golden ratios (1:1.618): the vertical distance between the chin and mouth vs that between the mouth and eyes (yellow arrows), and the vertical distance between the chin and that between the lower end of nose vs the lower end of nose and the hair line (green arrows). In this figure, 1.618 indicates the golden number, which is denoted by $\phi$ below.

In Figure 6, let $A, B, C$ and $D$ be the distances between adjacent red lines from top to bottom. Then the golden ratios are expressed as follows:

$$
\begin{aligned}
& (A+B):(C+D)=\phi: 1 \\
& (B+C): D=\phi: 1 .
\end{aligned}
$$

Let $H$ be the height of the face, and kept constant. Then we have

$$
A+B+C+D=H \text {. }
$$

From Htsui and Isaji's another result that the vertical length of a nose is about $28 \%$ of the height of the face for every face, we have

$$
B=0.28 H \text {. }
$$

The remaining $A, C$ and $D$ are given by solving (13), (14) and (15) simultaneously as follows:

$$
\begin{aligned}
A & =\frac{\phi}{\phi+1} H-B=\frac{0.72 \phi-0.28}{\phi+1} H \\
C & =\frac{A}{\phi+1}=\frac{0.72 \phi-0.28}{(\phi+1)^{2}} H \\
D & =\frac{B+C}{\phi}=\frac{0.28}{\phi+1} H .
\end{aligned}
$$

Figure 7 shows an example of this vertical transformation.

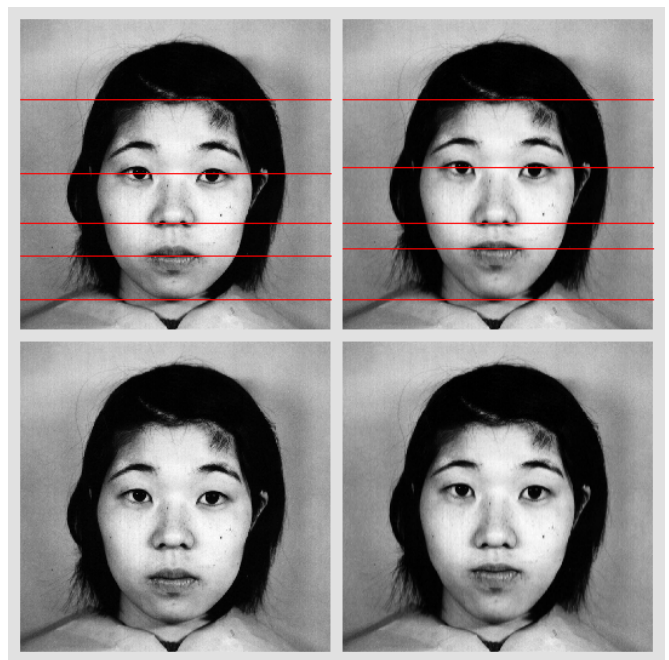

Figure 7: Vertical transformation based on the golden ratios in Figure 6 and Htsui and Isaji's result.

\section{Experimental Results}

In this section, we show the generated face images with the proposed methods described in the above section, and the results of the objective and subjective evaluations of the generated images.

3.1 Generated Face Images In this subsection, we demonstrate the generation of face images by the proposed three methods described Section 2.

Figure 8 shows three examples of generated face images for a single subject for comparing the change of the impression of the same subject, where Figures 8(a), (b) and (c) satisfy Conditions 1, 2 and 3 in Section 2.1, respectively. For Conditions 1 and 3 in Figures 8(a) and (c), the effects are small because these conditions are almost satisfied originally. On the other hand, Condition 2 in Figure 8(b) altered the impression very much as well as the result in Figure 2. Both results following Condition 2 have unnaturally pointed chins. To avoid this problem, it may be effective to handle the outline of face and the parts such as eyes, nose and mouth separately. 


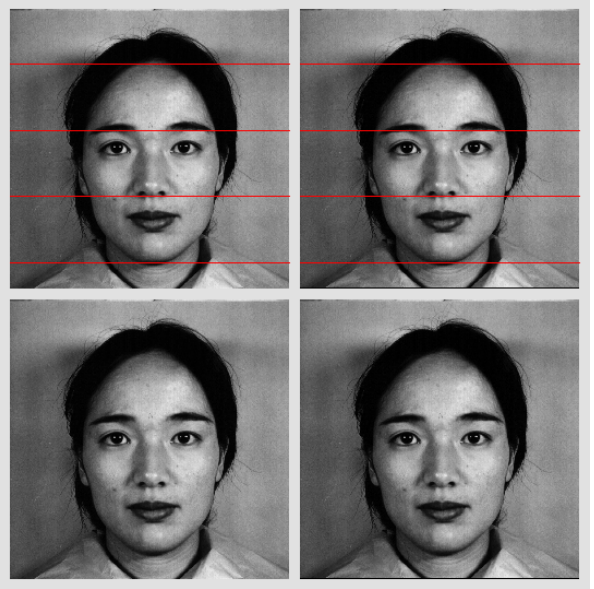

(a) Condition 1

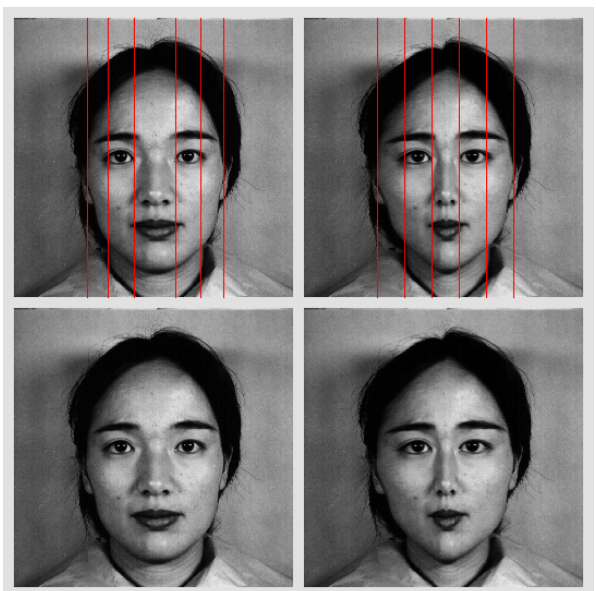

(b) Condition 2

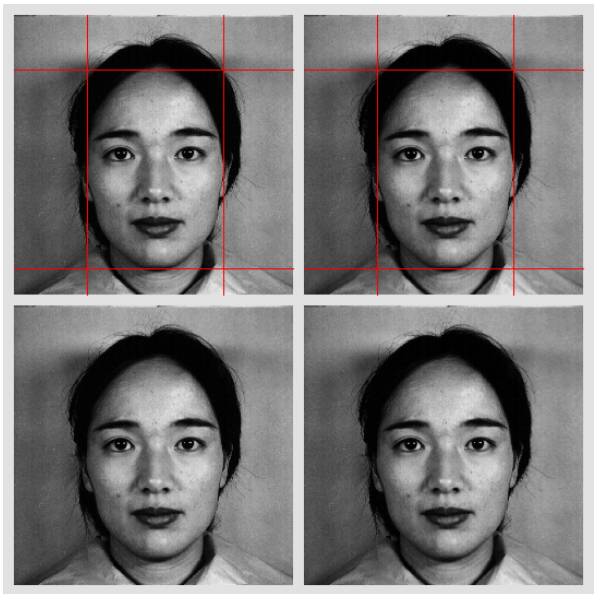

(c) Condition 3

Figure 8: Generation of face images satisfying Conditions 1, 2 and 3 presented in LAURIER PRESS [3] for the same subject image: (a) Condition 1, (b) Condition 2, and (c) Condition 3.

Figure 9 shows an example of the generation of a face image satisfying the three conditions in Section 2.1. In this example, although the vertical change caused by Condition 1 is small, Condition 2 makes the horizontal distance be-
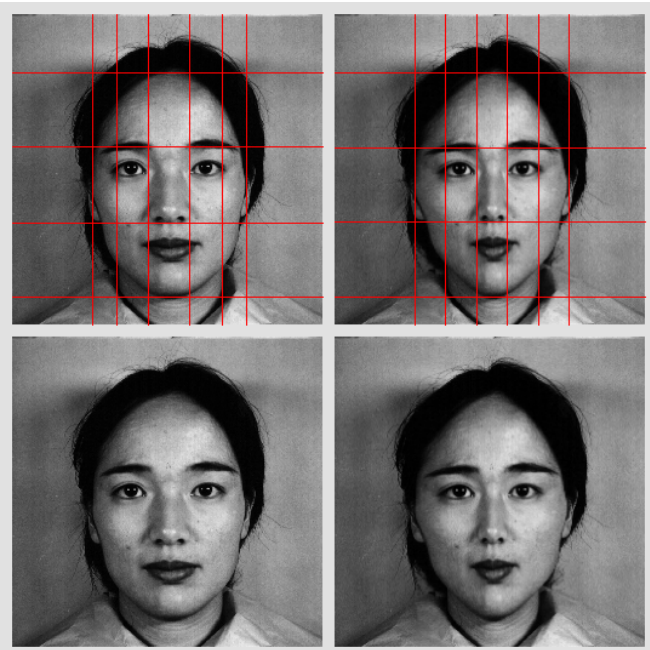

Figure 9: Generation of face image simultaneously satisfying Conditions 1, 2 and 3 presented in LAURIER PRESS [3].

tween eyes narrower, which enhances a factor of beautiful woman's face by Hatsui and Isaji [5]. Condition 3 is fulfilled originally in this example, and makes no major impact on the result.

Figure 10 shows an example of the generation of a face image based on Pallett's golden ratios described in Section 2.2, where the vertical distance between the eyes and

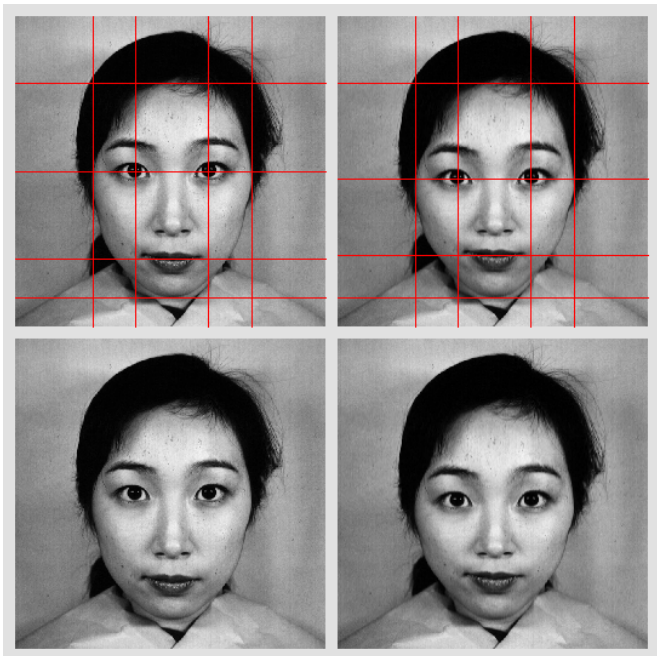

Figure 10: Generation of face image based on Pallett's golden ratios.

mouth gets narrower, and the resultant face becomes a kind of immature face.

Figure 11 shows an example of the generation of a face image based on Hatsui and Isaji's golden ratios and the vertical golden ratios in Figure 6 described in Section 2.3, where, in the horizontal direction, the widths of eyes expand, and the distance between the inner corners of both eyes gets narrower to get the factors of beautiful woman's face [5]. At the same time, the vertical transformation based on the mathematical golden ratios in Figure 6 makes the 


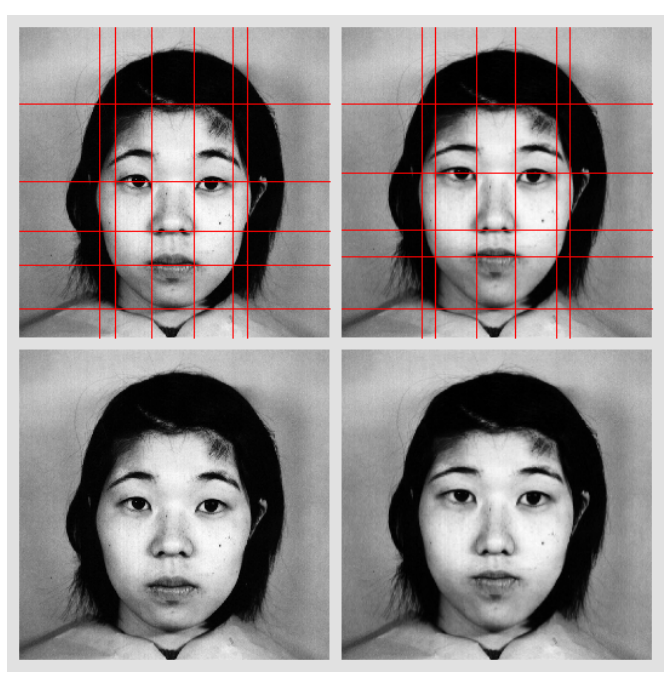

Figure 11: Generation of face image based on Hatsui and Isaji's golden ratios and that in Figure 6.

nose and chin longer.

3.2 Evaluations In this subsection, we evaluate the beauty of face images generated above objectively and subjectively.

Firstly, we show the result of objective evaluation by using a web application called Deeplooks [15]. Figure 12 shows the screenshots of Deeplooks. The left image shows
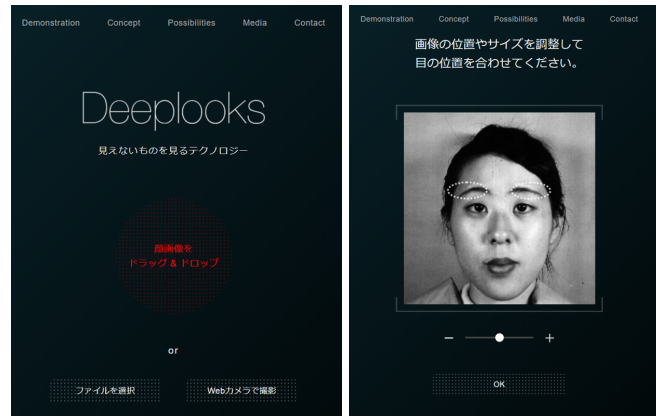

Figure 12: Deeplooks: If we drag \& drop a photo to the center of the homepage (left), then we proceed to the next stage (right), where we adjust the placement and size of the eyes according the guide.

the homepage to which we give a face image the beauty of which we want to evaluate. Then it switches to the right image which prompts us to adjust the eyes to two ellipses drawn with dotted lines. After the adjustment, we click OK to view the result as shown in Figure 13, where the transformed face image in Figure 3 is evaluated as 3.2-star rating (the maximum is 5 stars).

Figure 14 shows the scores of target face images, where the vertical and horizontal axes denote the score and the figure numbers in this paper, respectively. The blue and orange bars denote the original and generated face images. For 6 out of 8 faces, the scores were improved by the proposed method, and the mean score has increased from 2.76 to 2.91 .

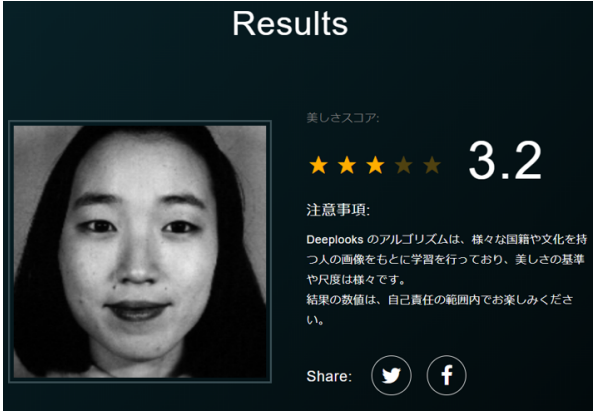

Figure 13: Evaluation result by Deeplooks.

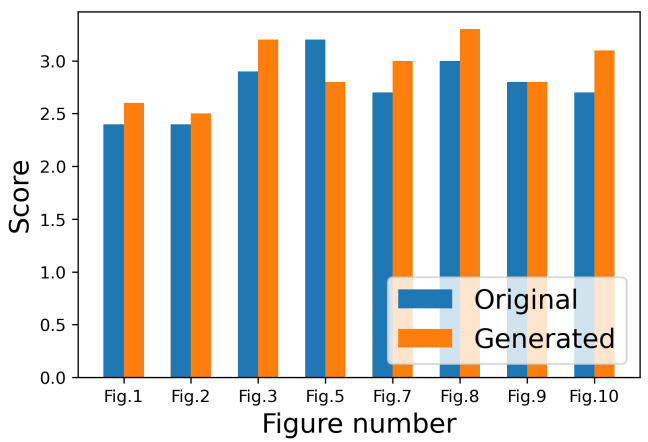

Figure 14: Scores given by Deeplooks.

Next, we show the result obtained by using another web application called Test for grading faces [16] (TGF). Figure 15 shows a screenshot of TGF, where all we need to do is to give a face image. In this figure, the transformed image in Figure 9 is given and evaluated as 62 out of 100 points, and additional information such as the estimated age and sex is also shown.
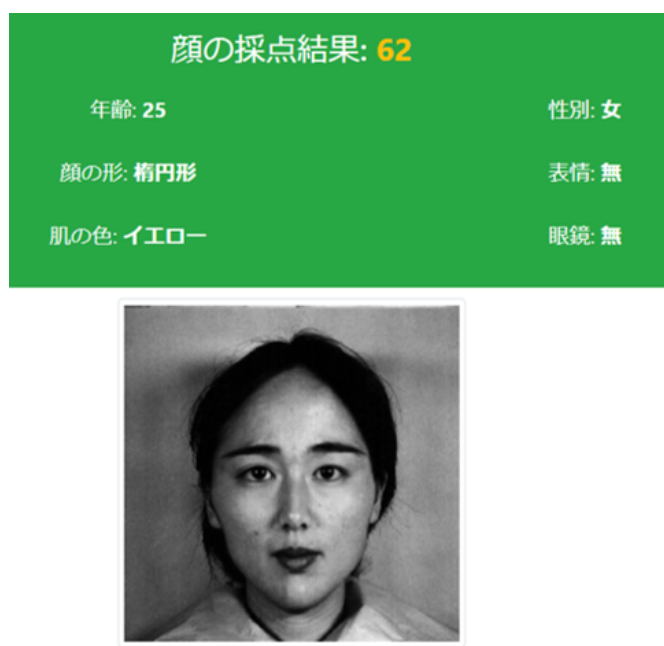

Figure 15: Evaluation result by TGF.

Figure 16 shows the scores of target face images, where, for the five faces among eight faces, the scores were improved by the proposed method, and the mean score has increased from 45 to 49 . For the transformed face image in Figure 10, although the score decreased from 50 to 49 , she became younger from 25 to 24 years old. 


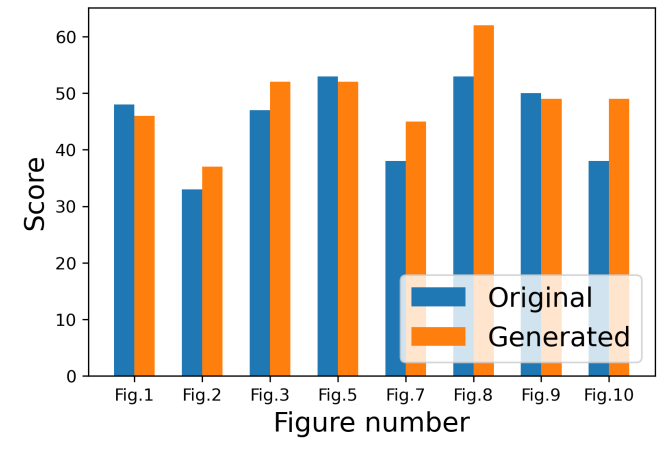

Figure 16: Scores given by TGF.

We also evaluated the beauty of the face images subjectively by administering a questionnaire on the Internet, where we got 45 respondents the details of which are 14 males and 31 females between the ages of 20 and 30. In this survey, the original and transformed face images are presented to each respondent simultaneously, and then he/she chooses preferable one. Figure 17 shows the result, where

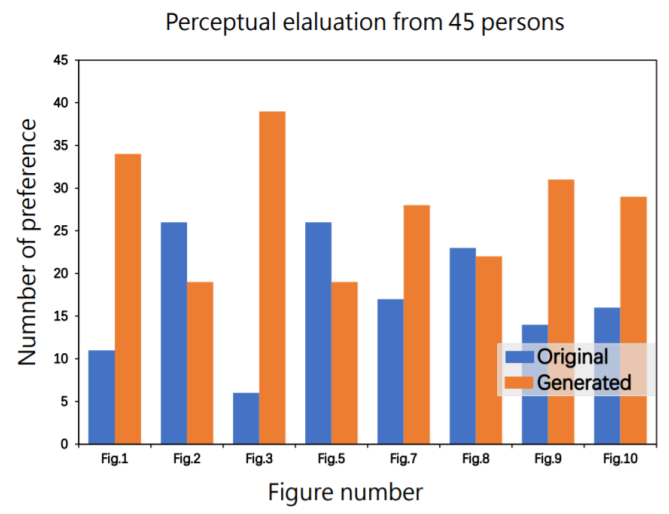

Figure 17: Result of questionnaire.

the vertical and horizontal axes denote the number of respondents who chose each face image as preferable one and figure numbers in this paper, respectively. The blue and orange bars denote the original and generated face images. For 5 out of 8 faces, the scores were improved by the proposed method, and the mean score has increased from 17.8 to 28.3. Consequently, we also verified the effectiveness of the proposed method subjectively, but the difference between the original and transformed face images was not statistically significant at the $5 \%$ significance level.

Table 1 summarizes the mean scores obtained above three evaluations: Deeplooks, TGF and the questionnaire, in which the generated face images by the proposed meth-

Table 1: Mean scores by three evaluation methods.

\begin{tabular}{c|ccc}
\hline & Deeplooks & TGF & Questionnaire \\
\hline Original & 2.76 & 45 & 17.8 \\
Generated & $\mathbf{2 . 9 1}$ & $\mathbf{4 9}$ & $\mathbf{2 8 . 3}$ \\
\hline
\end{tabular}

ods achieved higher mean scores in all evaluation methods.

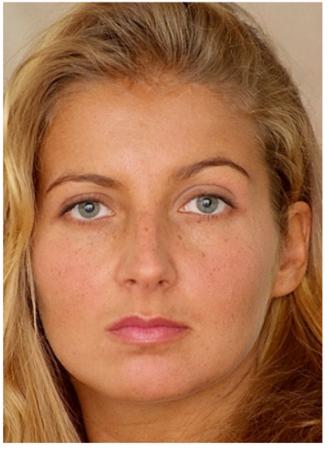

(a) Input

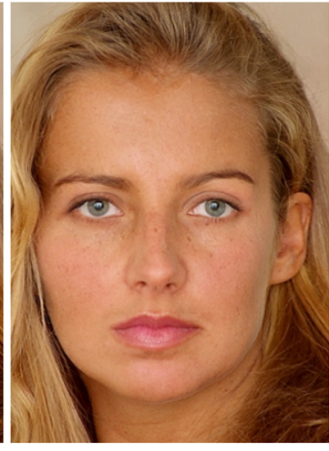

(b) Output
Figure 18: Result by Leyvand et al. [8].

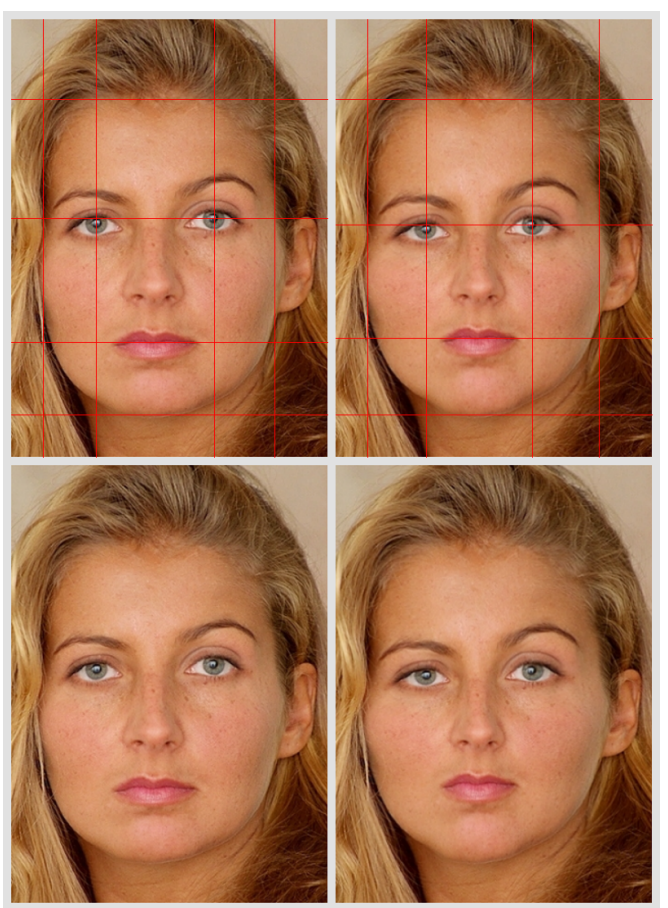

Figure 19: Result by the proposed method with Pallett's golden ratios.

Finally, we compare the proposed method with Leyvand's SVR-based beautifier [8]. Figure 18 shows an example presented by Leyvand et al. [8]. On the other hand, Figure 19 shows the result by the proposed method with Pallett's golden ratios described in Section 2.2. In this example, the vertical distance between eyes and mouth gets narrower, and the horizontal distance between eyes also gets narrower, that reduced the size of mouth too. As a result, we obtained a kind of immature face again.

\section{Conclusions}

In this paper, we proposed a method for transforming face images based on various golden ratios for facial beauty, and demonstrated the transformation of real face images by the proposed method with a publicly available face image dataset. We also evaluated the beauty of those faces both objectively and subjectively, and showed the effectiveness of the proposed method.

Our future work will include the improvement of the pro- 
posed method by incorporating facial symmetry, personal preference and mesh transform.

\section{Acknowledgment}

This work was supported by JSPS KAKENHI Grant Number JP21K11964.

\section{References}

[1] R. Paul, J. Sharrard, S. Xiong, "The Importance of Face-toFace Communication in the Digital World," Journal of Nutrition Education and Behavior, Vol. 48, No.10, p. 681, 2016. DOI: 10.1016/j.jneb.2016.09.014

[2] A. Laurentini and A. Bottino, "Computer analysis of face beauty: A survey," Computer Vision and Image Understanding, Vol.125, pp. 184-199, 2014. DOI: 10.1016/j.cviu.2014.04.006

[3] LAURIER PRESS. https://laurier.excite.co.jp/ i/HadaLove_121255, (Accessed date: 2021.2.1). In Japanese.

[4] P. Pallett, S. Link, and K. Lee, "New "golden" ratios for facial beauty," Vision Research, Vol. 50, No. 2, pp.149-154, 2010. DOI: 10.1016/j.visres.2009.11.003

[5] H. Hatsui and S. Isaji, "The factors of beautiful face in women's university student," Bull. Mukogawa Women's Univ. Nat. Sci., Vol.59, pp.1-6, 2011. In Japanese. DOI: 10.14993/00000509

[6] Japanese Academy of Facial Studies, "FaceTool". http: //nae-lab.org/project/face/IPA/, (Accessed date: 2021.2.1)

[7] Naemura Lab @ The Univ. of Tokyo, Tool for generating mean face. https://nae-lab.org/project/face/ HeikinTool/, (Accessed date: 2021.2.1)

[8] T. Leyvand, D. Cohen-Or, G. Dror, and D. Lischinski, "Data-driven enhancement of facial attractiveness," ACM Trans. on Graphics, Vol. 27, No.3, 2008. DOI: 10.1145/1399504.1360637

[9] N. Diamant, D. Zadok, C. Baskin, E. Schwartz, and A. Bronstein, "Beholder-gan: Generation and beautification of facial images with conditioning on their beauty level," in Proc. IEEE International Conference on Image Processing (ICIP), pp. 739-743, 2019. DOI: 10.1109/ICIP.2019.8803807

[10] T. Karras, T. Aila, S. Laine, and J. Lehtinen, "Progressive Growing of GANs for Improved Quality, Stability, and Variation," 6th International Conference on Learning Representations, ICLR, 2018. https://openreview.net/forum? id=Hk99zCeAb, (Accessed date: 2021.2.1)

[11] A. Martinez and R. Benavente, "The ar face database," CVC Technical Report, June 1998. http://www2.ece. ohio-state.edu/ aleix/ARdatabase.html, (Accessed date: 2021.2.1)

[12] Z. Liu, P. Luo, X. Wang and X. Tang, "Deep Learning Face Attributes in the Wild," 2015 IEEE International Conference on Computer Vision (ICCV), Santiago, Chile, 2015, pp. 3730-3738, DOI: 10.1109/ICCV.2015.425.
[13] M. Lyons, S. Akamatsu, M. Kamachi and J. Gyoba, "Coding facial expressions with Gabor wavelets," Proceedings Third IEEE International Conference on Automatic Face and Gesture Recognition, Nara, Japan, 1998, pp. 200-205, DOI: 10.1109/AFGR.1998.670949.

[14] E. Prokopakis, I. Vlastos, V. Picavet, G. N. Trenite, R. Thomas, C. Cingi, and P. Hellings, "The golden ratio in facial symmetry," Rhinology, Vol. 51, No.1, pp. 18-21, 2013. DOI: 10.4193/Rhino12.111

[15] "Deeplooks - a technology that visualizes the invisible," https: //deeplooks. com/ (Accessed on 08/03/2020).

[16] "Test for grading faces - Diagnosis of charm of appearance," https://beauty.doratool.com/ (Accessed on 08/03/2020). In Japanese.

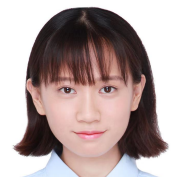

Ran Peng (Non-member) received B.S. degree from Dalian Minzu University of China in 2016. She started studying in Japan in 2018. She is currently a graduate student in Kyushu University. Her research interests include image enhancement and image processing.

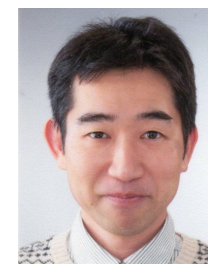

Kohei Inoue (Member) He received B.Des., M.Des. and D.Eng. degrees from Kyushu Institute of Design in 1996, 1998 and 2000, respectively. $\mathrm{He}$ is currently an Associate Professor in Kyushu University. His research interests include pattern recognition and image processing.

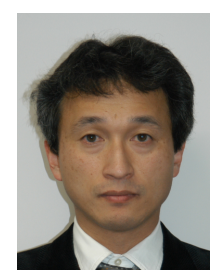

Kenji Hara (Member) He received the BE and ME degrees from Kyoto University in 1987 and 1989 , respectively, and the $\mathrm{PhD}$ degree from Kyushu University in 1999. He is currently a Professor in Kyushu University. His research interests include physics-based vision and geometric modeling. 